\title{
"Deep intubation technique for Complex Percutaneous Coronary Intervention. Insights of a feared and harmless technique"
}

\author{
Ricardo Allende ${ }^{1}$, Gérald Barbeau ${ }^{2}$, Josep Rodés-Cabau ${ }^{2}$ \\ ${ }^{1}$ Hospital de Especialidades Médicas de la Salud. San Luis Potosí, S.L.P. Mexico. \\ ${ }^{2}$ Quebec Heart and Lung Institute. Quebec city, QC. Canada.
}

\begin{abstract}
:
Background: A successful percutaneous coronary intervention (PCI) on complex coronary anatomy can be up to $90 \%$ of cases and depends on many factors such as successfully guiding catheter support, which entails a perfect entry in the ostium of the treated artery, the guidewire's ability to cross the culprit lesion and the stent profile. In some transradial artery cases the vessel's diameter does not allow the use of a thick guiding catheter (from 6 to $8 \mathrm{Fr}$ ), in these cases special techniques such as "deep intubation" may be useful, if performed correctly.
\end{abstract}

Methods: We describe four different percutaneous coronary interventions (PCI) scenarios related to stent delivery in difficult procedures involving saphenous vein graft, mammary artery graft and native coronary arteries in complex anatomies with no incurring complications.

Results: Interestingly, deep-intubation has been considered a dangerous and contraindicated procedure to perform through coronary angioplasty, nonetheless we found that in many cases the deep intubation technique is an effective and convenient procedure when performed with small sized catheters at low cost. 


\section{Introduction}

A successful complex percutaneous coronary intervention (PCI) relies on different items such as tortuosity, calcification and the severity of the lesion. Its performing might be associated to some serious and life-threatening complications such as coronary dissection, vessel perforation, stent loss, or twisting and tangling of the guidewires to mention some. ${ }^{1}$ PCI has some characteristics to being safely be completed. The Guiding catheter's (GC) support. The guidewire's force to cross the culprit lesion and to advance the stent. And finaly, the stent's profile, its flexibility and pushability may ease or complicate the procedure.

Out of these factors, catheter's support is the only one that could be modified by changing position on the coronary ostia and the aorta. Passive support of the catheter is given by its conformation, its thickness and the material it is made of. Active support, on the other hand, is given specifically by the operator, which implies the cannulation technique of the artery, its depth and the approach on the vessels ostiujm or the help provided by the guidewire, ${ }^{2}$ anchoring balloon technique, or the deep intubation technique. ${ }^{3} 4$ The telescoping approach with a "mother and child" technique might be useful but sometimes twisting of the guidewire could present. ${ }^{5,6}$ The use of microcatheters on complex PCI might also increase success angioplasty. ${ }^{7}$

Most of these techniques require a 6-French catheter or larger, which is sometimes difficult to use by radial approach. Some of the limitations using 6-French or larger catheter include the tortuosity, spasm and vessel caliber that increases vascular complications, dissection and rupture. A painful and laborious procedure with longer fluoroscopy and radiation exposure to both the patient and operators. Radial artery thrombosis and dissections are both complications that present on patients who have suffered from spasm and it is more frequent with a $>1$ sheath/artery ratio. ${ }^{8}$ Although effective, procedural cost may increase with some of these techniques, having the deep intubation technique as one of the most effective with low cost when carefully performed. ${ }^{9}$

\section{Methods}

We performed a review of the literature of the deep intubation technique and other procedures which imply the introduction of a catheter within at least $20 \mathrm{~mm}$ further to the ostium. Procedural success, complications and tips and tricks to complete this technique are discussed and compared to four different scenarios using this approach. 


\section{Review of the literature}

Complex PCI is generally treated with a 6 Fr catheter; however, the use of a 5 Fr catheter might allow the deep-intubation technique, which has the benefit of being low cost, requiring no extra equipment and being compatible in most patients. ${ }^{10}$ By definition, the deep intubation technique is the advancing of the guiding catheter $2 \mathrm{~cm}$ or deeper into the native artery. ${ }^{11}$ Most centers and recommendations suggest not to use this technique, nevertheless, some retrospective studies have shown that up to $2.6 \%$ of coronary complex procedures have required this technique. ${ }^{12}$ Despite, this method might be risky if performed by an unexperienced operator. We share the procedure used on each one of four different difficult scenarios using the deep intubation method, and their advantages on complex situations.

\section{The deep intubation technique using a 5 French catheter}

To perform a successful and low-risk "deep intubation technique" the guiding catheter must be well cannulated to the ostium of the desired artery whilst previously locating the lesion site. The 5 French is recommended due to its low complications when compared to 6 French or larger devices when examined by optical coherence tomography (OCT). ${ }^{13}$ No changing to larger catheters has been needed when properly performed. ${ }^{14}$ A careful advance of the guidewire as far as possible within the artery is performed to provide optimal support. A balloon no larger than the vessel's size is then advanced down to the desired lesion or as close as possible and is inflated at low pressures (6-8 atm), then the guiding catheter is carefully slid into the artery using the balloon as an "anchoring balloon". No contrast injection is recommended up to this moment. Some authors have reported inflating a balloon on the tip of the catheter to prevent from dissection the artery mimicking "proboscides".

After having advanced the catheter, the stent may be carefully advanced into the lesion, noting that too much pressure into de stent might push the catheter out of the artery. Deflation and withdrawal of the anchoring balloon is performed with posterior advancing of the proper stent to the desired lesion. After having advanced the stent the catheter is drawn back to the ostium and proper positioning of the stent might be confirmed. As of this moment, little contrast injection is allowed and the angioplasty may be performed. A final contrast injection is always required with normal vessel characteristics, full filling of the artery and no residual contrast staining site must be observed. In case of narrowing of the arterial diameter a repeated injection with vasodilators such as nitroglycerin could be of use. In the approach of the deep intubation technique some aspects must be carefully considered on all occasions: 
- Careful arterial pressure surveillance must be carried on without exception, and the pressure curve should be within acceptable parameters. A reduction in the invasive blood pressure curve with ventricularisation (low diagnostic values) indicates that the catheter is obstructive.

- When the pressure curve loses its normal shape and resembles the pulse pressure, it could mean the catheter is obstructing the vessel flow and must be retrieved as soon as possible. ${ }^{15}$

- Contrast filling of the artery is to be homogeneous and without flares which might indicate the presence of dissections secondary to the use of the sent or catheter. Contrast injection should be done cautiously in order not to suffocate the artery. If the contrast staining the artery a dissection must be considered. At the same time, normal flow of contrast needs always to be seen; if stagnation of flow is seen withdrawal of the catheter should be performed until normal passing of blood is confirmed.

- Basal rhythm of the patient must be always present. Slowing-down of the basal heart rate should make the operator suspect flow obstruction. Morphology of the electrocardiogram and chances in rhythms might be an omen of possible arrhythmias due to flow changes. If seen, withdrawal of the catheter should be done until basal rhythm returns.

- $\quad$ Chest pain or angina might suggest catheter- related blood-blockage. However as with many coronary angioplasties, pain might be present while balloon inflation is performed.

\section{The deep intubation technique using a mother-and-child catheter.}

The mother-and-child system (also known as catheter extension or transit catheters) is a technique in which a small and flexible catheter is introduced within a larger system to achieve a more rigid support while performing complex angioplasties with good results. ${ }^{16}$ The extension catheter has some advantages:

- To have continuous pressure measurement without obstructing blood flow on most vessels.

- $\quad$ The tip of the catheter is a low-traumatic device that could be inserted up to several $\mathrm{mm}$ inside the culprit artery with low risk of dissection.

- Allows the mother catheter to be moved outside the coronary ostium and allow the artery to "breathe or receive more blood flow" while dilating.

- $\quad$ Facilitates the placement of interventional devices 
- Allows to advance the balloon or stent down to the culprit lesion and then to pull back the child catheter inside the mother to perform different angiographies before dilating without obstructing while providing additional guide back-up support.

The extension-catheter consists of a $120 \mathrm{~cm}$ proximal hypotube shaft with a $25 \mathrm{~cm}$ from 6 to 8 French catheter and a distal hydrophilic coating. ${ }^{17}$

The mother catheter is inserted into the coronary ostium, a standard angiography injection is performed always revising the blood pressure curve. If no damping is suspected, the 0.014" guidewire is carefully advanced through the culprit lesion. Then, the child catheter is purged with normal saline $0.9 \%$ or sterile water, and the distal part of the catheter is inserted as an over the wire device and introduced into the mother catheter and pushed within the lumen close to the tip tip of the catheter. It is very important to make sure that no bubbles are within the system when inserting. Having performed this part, the child-catheter is slowly advanced through culprit vessel down to the desired section of the artery before the target lesion. After that, the stent or balloon is inserted into the angioplasty guidewire and pushed into the catheter, while advancing, fluoroscopy should be performed when the balloon or stent arrives to the distal part of the extension catheter so that when inserted, no damage to it would occur to the tip of the balloon. Later, the balloon is advanced as a regular angioplasty and the procedure is completed as usual. (Figure 1)

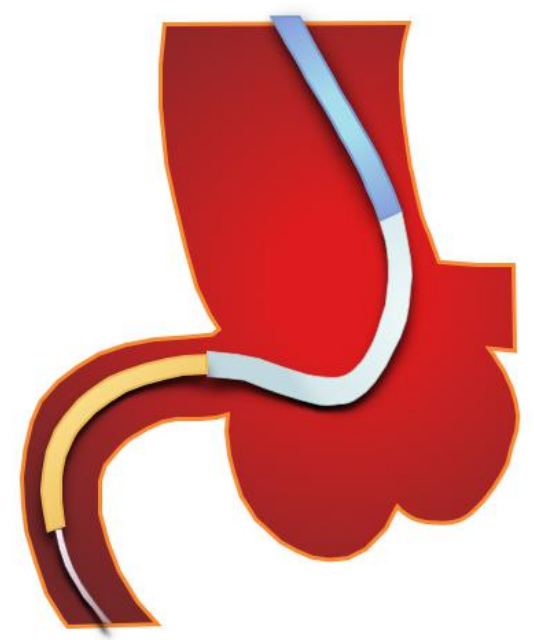

Figure 1. Schematic representation of the catheter extension on the right coronary artery. (Guidezilla ${ }^{\mathrm{TM}}$, Boston Scientific, Natick, Masachussets, USA) Notice the advancing of the 0.014 " guidewire into the distal portion of the vessel with the catheter extension (yellow) coming out from the guiding catheter (blue).

After the stent is placed and dilated, the child catheter is pulled back into the guiding catheter carefully holding the angioplasty guidewire to prevent from retrieving it from the vessel. A 
control angiography should be performed and If no complications are seen, the guidewire could be withdrawn and the system is to be taken out.

If residual lesion, dissection or another plaque is discovered, the system ought to be carefully readvanced and a new angioplasty could be accomplished.

\section{Patients and methods.}

We performed angioplasties on four different complex cases, in which deep intubation technique was required to achieve a successful percutaneous coronary intervention using only a 5 Fr catheter.

\section{Case 1.}

A 75-year-old man was admitted to the hospital due to new-onset angina. His past medical history included a coronary artery bypass graft (CABG) 23 years earlier for left-main and three vessel disease. He had a left internal mammary artery (LIMA) graft anastomosis to left anterior descending (LAD) as well as a saphenous vein graft (SVG) to the right coronary artery (RCA) and obtuse marginal (OM).

A coronary and graft angiography was performed which showed $100 \%$ left main occlusion, $70 \%$ proximal and $90 \%$ distal LAD lesions as well as a completely occluded circumflex artery (Cx). The saphenous vein graft to RCA was completely occluded with a patent saphenous graft to $\mathrm{Cx}$ and patent LIMA to LAD. Finally, a Rentrop II collateral circulation from the LAD to the RCA was observed.
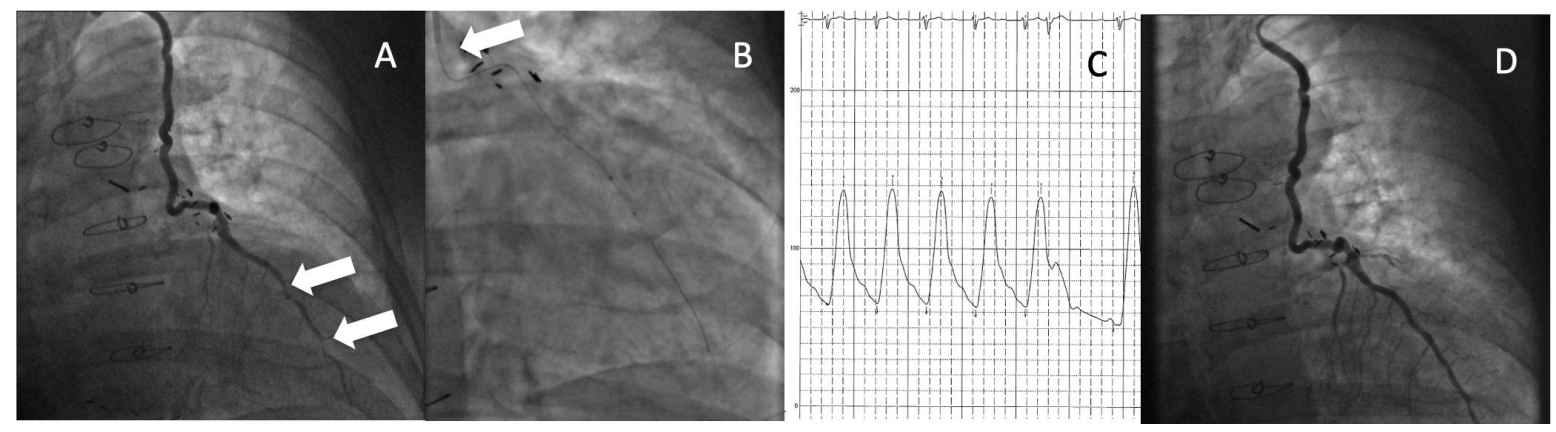

Figure 2. Deep intubation on arterial graft to left anterior descending artery. (A) Angiography of left internal mammary artery (LIMA) performed by left radial artery approach showing significant lesions on the mid and distal portion of the left anterior descending artery (LAD) (B) Image showing the tip of a LIMA catheter pointed by the arrow while performing the deep intubation technique. (C) Normal arterial pressure curves while performing the deep intubation technique with the 5F LIMA catheter. (D) Final result after performing successfully the deep intubation technique with no traces of artery dissection.

Due to the chronically occluded native left main, RCA and its venous graft as well as the fact that the LAD was the main blood supply of the anterior and inferior portions of the heart, an 
angioplasty of the native left anterior descendent artery was programmed through the LIMA graft. (Figure 2-A)

Different approaches, balloons and 0.014" guidewires were used to cross the lesion with no success because of the lack of support. Nevertheless, a Cordis modified 5 Fr LIMA catheter (Cordis Corporation, Fremont, California) was then used with better support using the deep intubation technique by descending through the distal internal mammary artery and subsequently Whisper MS guidewire (Abbott Laboratories, Chicago, Illinois) was distally advanced toward the lesions. (Figure 2-B). Predilation of the lesions was accomplished with a Mini Trek 2.0 x 12mm (Abbott Laboratories, Chicago, Illinois) with the posterior deployment of two Promus Element Stents (Boston Scientific Corp, Natick, Massachusetts). During the procedure, careful surveillance of the pressure-wave curve was registered with no observed variations (Figure 2-C) Final angiography showed excellent distal T1M1 3 flow and no dissections in the LMA graft. (Figure 2-D).

\section{Case 2.}

An 82-year-old woman was admitted after being diagnosed with a Non-ST elevation myocardial infraction (NSTEMI). The diagnostic coronary angiography showed $90 \%$ ostial LAD lesion, $100 \%$ ostial Cx lesion and an $80 \%$ lesion on distal RCA which caused collateral circulation to the second marginal artery. A surgical revascularization treatment was considered and posteriorly rejected because of the high risk it entailed, finding a percutaneous approach to be more appropriate. The angioplasty of the left main artery and LAD was successfully performed without any complications. Afterwards we sought to conduct and angioplasty of the RCA where it was complicated to advance the stent because of the vessel's diffuse atherosclerosis, calcification and tortuosity, which along with the lack of support made it difficult to cannulate the vessel. A 5 Fr Barbeau catheter (Boston scientific Corporation, Natick, Massachusetts) was deeply intubated into the mid RCA using an 0.014" Choice PT guidewire (Boston Scientific Corp, Natick, Massachusetts) and a 2.75 x $38 \mathrm{~mm}$ Promus Element stent was distally delivered (Boston Scientific Corp, Natick, Massachusetts) (Figures 3-A and 3-B) while maintaining adequate arterial blood pressure throughout de procedure. After the stent delivery, a control angiography was performed with no arterial dissection and good angioplasty results. (Figure 3-C) 


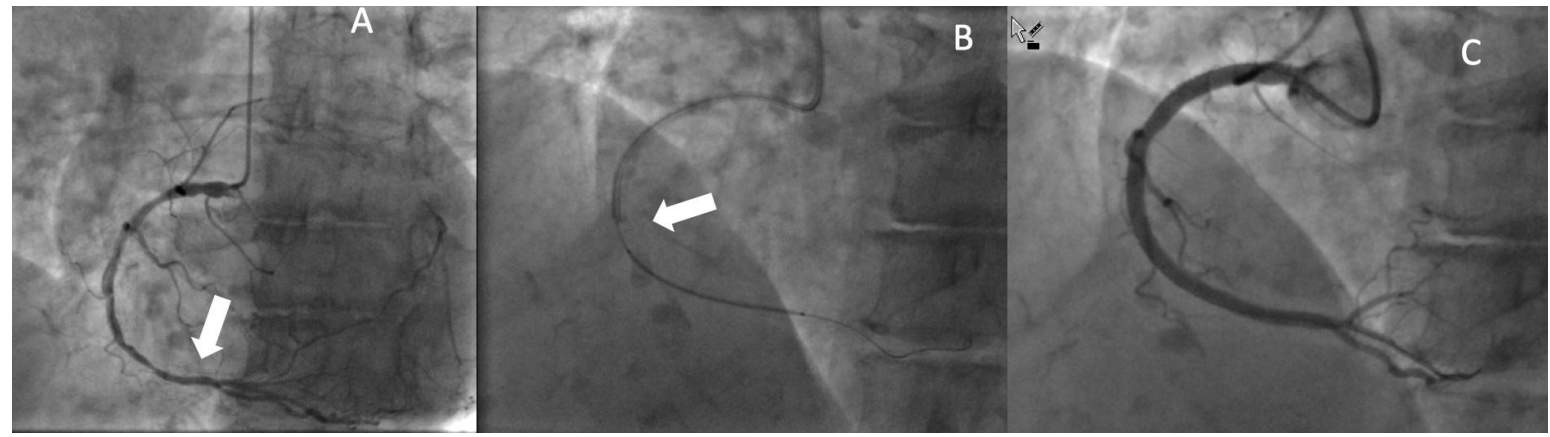

Figure 3. Deep intubation on native right coronary artery. (A) Right coronary artery with diffuse lesions and a severe obstruction pointed by the arrow. (B) Image showing the tip of the catheter while performing the deep intubation technique with a $5 \mathrm{~F}$ Barbeau guiding catheter. (C) Final results after performing successfully the deep intubation technique.

\section{Case 3.}

An 81-year-old male presented with an acute coronary syndrome. His medical history included a 33-year-old diagnosis of CABG that required using saphenous vein grafts to the LAD, left posterior descending (LPD) and a jump graft to the first and second marginal arteries. Nineteen years after his initial CABG he underwent a bypass surgery from LIMA to LAD. Additionally, he suffered two ACS, the first one was treated medically and the second was treated with a PCI of the venous graft to the second marginal.

During his hospitalization from the acute coronary syndrome the coronary angiography showed $100 \%$ lesions of the left main and RCA. There was no obstruction from his LIMA to LAD. The venous graft to the LPD has an $80 \%$ distal lesion. The proximal stent (which was deployed the previous year) in his venous graft to the first and second marginal was patent but a new $90 \%$ lesion was discovered in the mid portion of the vessel. (Figures 4-A and 4-B). Because of the patient's high surgical risk a percutaneous approach was chosen. Angioplasty of both venous bypass grafts was performed using a 5 Fr Barbeau Catheter (Boston Scientific Corporation, Natick, Massachusetts) and a 0.014" balance middleweight universal guidewire. (Abbott Laboratories, Chicago, Illinois). The attempt to advance a balloon into the distal portion of the graft was unsuccessful because of poor catheter support, therefore a cautious deep intubation of the graft was conducted to the middle portion allowing the delivery and successful stenting of the graft using a Multi-link 3.5 × $38 \mathrm{~mm}$ stent. (Abbott Laboratories, Chicago, Illinois) (Figure 4-C). No complication was observed on final angiography and a fully treated graft with distal flow was obtained. (Figure 4-D) 


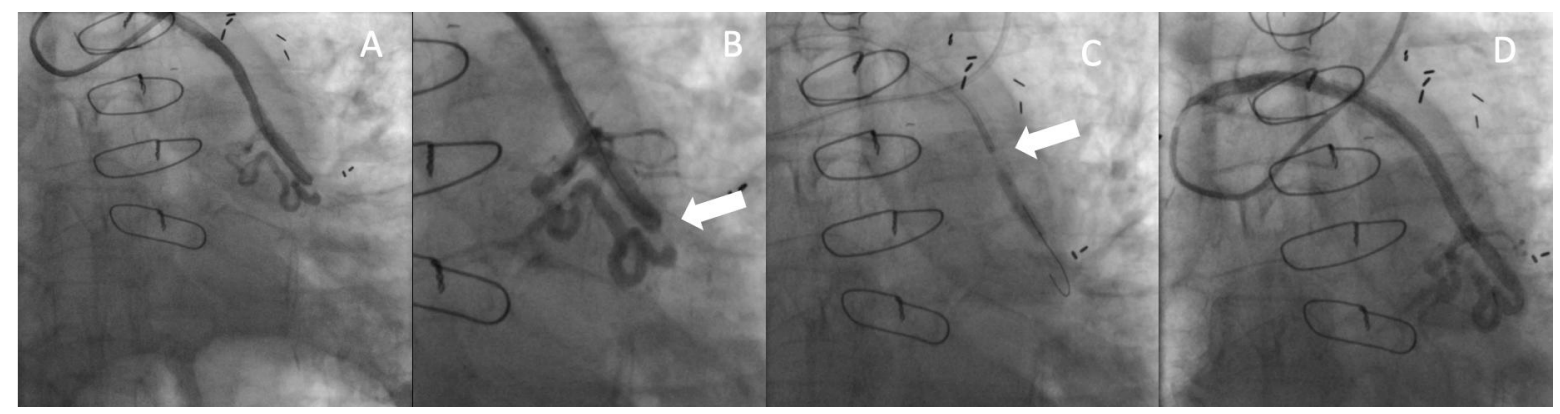

Figure 4. Deep intubation on vein graft to marginal artery. (A) Anteroposterior angiography of saphenous vein graft with a severe lesion on the anastomosis portion to a marginal artery. (B) Amplified image highlighting the portion of the vein graft with a severe lesion on the anastomosis to the coronary artery. (C) Tip of the catheter performing deep intubation while performing the anchoring technique with the inflated balloon proximal to the lesion performed by a $5 \mathrm{~F}$ multipurpose guiding catheter. (D) Image showing the result after performing the angioplasty successfully.

\section{Case 4.}

Sixty-year-old male with history of CABG in 1987 using a saphenous vein graft to the LPD and a LIMA to LAD. He presented in early 2011 with chest pain, requiring an angioplasty of the distal left main and proximal Cx. In late 2011, he suffered a NSTEMI where an Everolimus stent was used to correct an in-stent restenosis of the Cx.

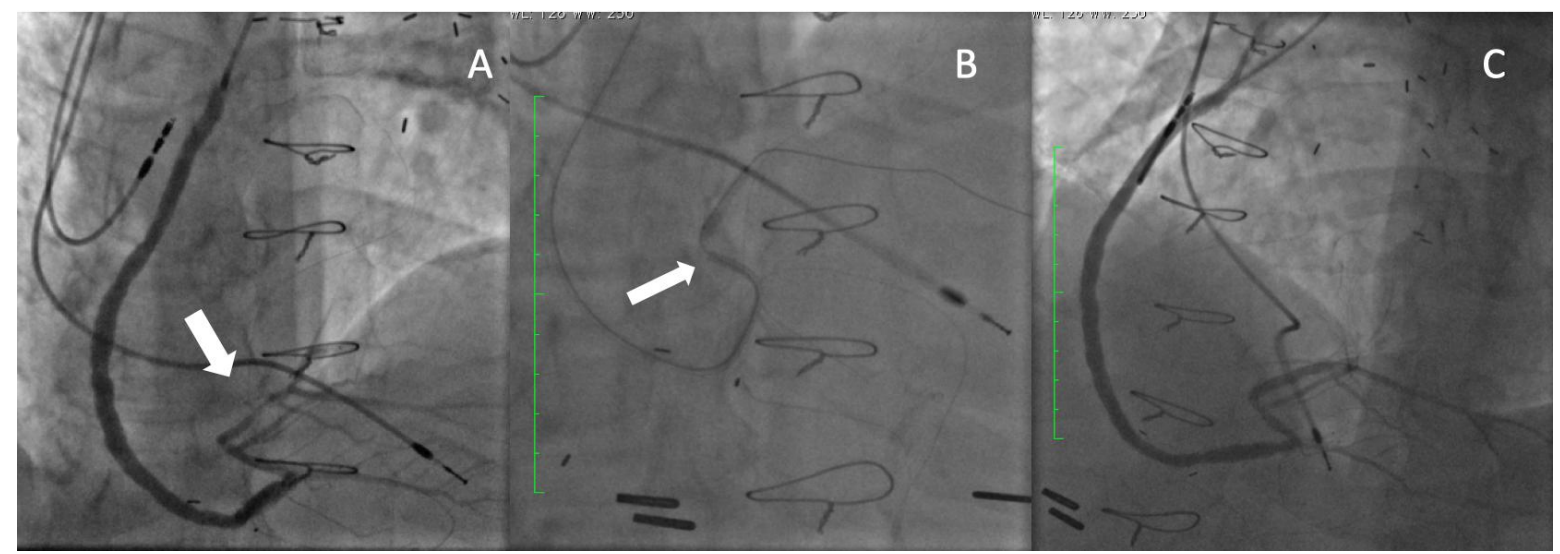

Figure 5. Deep intubation on vein graft to left posterior descending artery. (A) Angiography which shows distal left posterior descending artery lesion distal to the anastomosis on a very tortuous artery. (B) Image which showing deep intubation while performing the anchoring technique with a $5 \mathrm{~F}$ multipurpose guiding catheter. (C) Angiography showing result with permeability of the stent and no damage on the graft.

In April 2013, he was admitted for unstable angina. His angiography showed patent left main and Cx stents, a patent LIMA, however the vein graft to the LPD had a new lesion on its distal portion, extending to the anastomotic site and tortuous native vessel. (Figure 5-A) A 5 Fr Barbeau catheter (Boston scientific Corporation, Natick, Massachusetts) was used to cannulate the graft and a Choice PT guidewire 0.014" (Boston scientific Corporation, Natick, Massachusetts) was advanced across the lesion. A major difficulty was encountered, as we were unable to deliver a balloon through the tortuous native vessel. Very distal intubation of 
the graft was achieved, while maintaining excellent arterial pressure curve and an Apex-Push balloon 1.5 x $15 \mathrm{~mm}$ (Boston Scientific Corporation, Natick, Massachusetts) was used to predilate the lesion. (Figure 5-B) After multiple balloon inflations a Resolute Integrity 2.5 x 26 mm (Medtronic Inc, Minneapolis, Minnesota) was successfully delivered. (Figure 5-C) A final injection was performed showing a patent native artery with no complications from deep intubation.

\section{Discussion}

Choosing the suitable equipment and performing the correct technique could make the difference in time, radiation, risk, introduction of special devices such as rotablator and above all, the success of a procedure. ${ }^{18} 19$ Even in some cases it has been used as a successful thromboaspiration device. ${ }^{20}$ Several techniques have been successfully developed to perform complex coronary artery stenting that require high catheter support, however, as is the case with most medical procedures, these techniques are not exempt from disadvantages.

The mother in child technique could be a plausible option but it often requires modification of the guiding catheter and the risk for an air embolism is latent.

The anchoring balloon allows to use a distal inflated balloon as a fixing point to advance the tip of the catheter into the artery, nevertheless, sometimes the artery is damaged at the point where the balloon was expanded and on other occasions, some cases of guidewire rupture have been reported.

The Proxis catheter minimizes the MACE however, it is considered an expensive device.

The Tornus catheter is a device that could be used to cross difficult lesions, but it is expensive and requires training and expertise to be used, no anterograde visualization is available and once removed, advancing of the stent to the lesions with the same support is needed. ${ }^{21}$

All the previously mentioned techniques, although useful, require a $6 \mathrm{Fr}$ at the very least, which is not always possible to use. It has been shown that a $6 \mathrm{Fr}$ sheath has an external diameter of $2.52 \mathrm{~mm}$ and only $86 \%$ of men and $73 \%$ of women have a larger diameter than the external one of the sheath. ${ }^{8}$

The deep intubation technique has proven to be a successful method with a low complication rate and satisfactory results. ${ }^{22}$ When performed with more than $20 \mathrm{~mm}$ of vessel intubation, procedural success might increase up to $100 \% .{ }^{23}$ It may be useful in cases where the stent 
delivery is difficult because of the presence of calcified and /or tortuous arteries during a percutaneous coronary intervention. ${ }^{12}$ When not performed correctly, this technique could result in coronary spasm, extensive dissections, arrhythmias or even cardiac arrest that may be difficult to manage. ${ }^{24,25}$ The principle of deep intubation has been used by other devices such as: extension catheters, microcatheters and mother-in-child technique to increase backup support. ${ }^{26}$ Added to this, contrast use has been demonstrated to be much less due to optimal vessel opacification to decrease the risk of contrast induced nephropathy. ${ }^{27}$

Previous reports have described the use of the telescopic microcatheters or catheter extension such as the Guideliner TM (Vascular solutions, Inc. Minneapolis, Minnesota) or Guidezilla TM (Boston Scientific, Natick, Massachusetts) in challenging PCI cases, ${ }^{26,28}$ The importance of appropriate support is seen with the high use of extension catheters in up to $97 \%$ of complex cases. However, air embolism and stent dislodgement have been registered with these devices as well as pressure dampening in as much as $57 \%$ of cases. ${ }^{17} 29$

Some authors have successfully treated lesions on the LAD through native internal mammary arteries using the Guideliner. ${ }^{30} 31$ No deep intubation technique on the LIMA graft has been reported. This strategy might help treat significant distal and difficult to treat lesion while avoiding iatrogenic dissections.

Some authors have reported the "five in seven" or the "five in six" technique but cannulation with a more robust catheter could end in vessel complications and fatal results. ${ }^{32}$

Our goal is not to minimize the use of other devices or techniques, but to clarify and show the proper use and success of the "deep intubation technique", emphasizing on its low cost and efficacy when performing coronary artery interventions.

\section{Conclusion}

The "deep intubation technique" if managed properly and with caution, is a safe and effective technique to perform coronary artery stenting where high support is required.

On patients who undergo a radial procedure and who possess difficult anatomy, the use of a 5 Fr catheter might allow for a coronary intervention despite the vessel's diameter since it increases malleability to perform safe coronary cannulation. Conversely it avoids friction of the stent along the vessel wall lowering the risk of vessel and stent damage. It lowers costs, time, radiation and the use of contrast. 


\section{References}

[1] Saeed B, Banerjee S, Brilakis ES. Percutaneous coronary intervention in tortuous coronary arteries: associated complications and strategies to improve success. J Interv Cardiol 2008;21:504-11.

[2] Jafary FH. When one won't do it, use two-double "buddy" wiring to facilitate stent advancement across a highly calcified artery. Catheter Cardiovasc Interv 2006;67:721-3.

[3] Fujita S, Tamai H, Kyo E, et al. New technique for superior guiding catheter support during advancement of a balloon in coronary angioplasty: the anchor technique. Catheter Cardiovasc Interv 2003;59:482-8.

[4] Zhang S, Xu K, Yang N, Li C. Anchor balloons assisted deep intubation of 5F catheters for uncrossable lesions. Niger J Clin Pract 2016;19:421-5.

[5] Hashimoto S, Takahashi A, Yamada T, et al. Spontaneous rotation of the monorail-type guide extension support catheter during advancement of a curved guiding catheter: the potential hazard of twisting with the coronary guidewire. Cardiovasc Interv Ther 2018;33:379-83.

[6] Ashikaga T, Sakurai K, Satoh Y, et al. Mother-child technique using a novel 4 Fr inner catheter. EuroIntervention 2015;10:1432-9.

[7] Takahashi S, Saito S, Tanaka S, et al. New method to increase a backup support of a 6 French guiding coronary catheter. Catheter Cardiovasc Interv 2004;63:452-6.

[8] Kotowycz MA, Dzavik V. Radial artery patency after transradial catheterization. Circ Cardiovasc Interv 2012;5:127-33.

[9] Aznaouridis K, Bonou M, Masoura K, Vaina S, Vlachopoulos C, Tousoulis D. Successful Stent Delivery Through a Slaloming Coronary Path. J Invasive Cardiol 2019;31:E43.

[10] Bagur R, Gleeton O, Rinfret S, De Larochelliere R, Bertrand OF, Rodes-Cabau J. Transradial extra deep coronary intubation with a guide catheter for direct thromboaspiration in acute myocardial infarction. Int J Cardiol 2012;158:e32-4.

[11] Peels HO, van Boven AJ, den Heijer P, Tio RA, Lie KI, Crijns HJ. Deep seating of six French guiding catheters for delivery of new Palmaz-Schatz stents. Cathet Cardiovasc Diagn 1996;38:210-3.

[12] Bartorelli AL, Lavarra F, Trabattoni D, et al. Successful stent delivery with deep seating of 6 French guiding catheters in difficult coronary anatomy. Catheter Cardiovasc Interv 1999;48:279-84. 
[13] Barlis P, Gonzalo N, Di Mario C, et al. A multicentre evaluation of the safety of intracoronary optical coherence tomography. EuroIntervention 2009;5:90-5.

[14] Garcia E, Moreno R, Gomez-Recio M. Successful stent delivery through 5 French guiding catheter. J Invasive Cardiol 2001;13:16-8.

[15] Di Mario C, Sutaria N. Coronary angiography in the angioplasty era: projections with a meaning. Heart 2005;91:968-76.

[16] Kumar S, Gorog DA, Secco GG, Di Mario C, Kukreja N. The GuideLiner "child" catheter for percutaneous coronary intervention - early clinical experience. J Invasive Cardiol 2010;22:495-8.

[17] de Man FH, Tandjung K, Hartmann M, et al. Usefulness and safety of the GuideLiner catheter to enhance intubation and support of guide catheters: insights from the Twente GuideLiner registry. EuroIntervention 2012;8:336-44.

[18] Nikolsky E, Gruberg L, Pechersky S, et al. Stent deployment failure: reasons, implications, and short- and long-term outcomes. Catheter Cardiovasc Interv 2003;59:324-8.

[19] Latsios G, Toutouzas K, Karanasos A, Tousoulis D. Use of Extra Deep Guide-Catheter Intubation for Rotablation-Facilitated Percutaneous Coronary Intervention of the Right Coronary Artery. Cardiovasc Revasc Med 2019;20:13-4.

[20] Moretti A, Sanz Sanchez J, Petriello G, Gasparini GL. Ultra-Deep Guide Catheter Intubation for Direct Thromboaspiration in Acute Myocardial Infarction. Cardiovasc Revasc Med 2019;20:49-50.

[21] Di Mario C, Ramasami N. Techniques to enhance guide catheter support. Catheter Cardiovasc Interv 2008;72:505-12.

[22] Von Sohsten R, Oz R, Marone G, McCormick DJ. Deep Intubation of 6 French Guiding Catheters for Transradial Coronary Interventions. J Invasive Cardiol 1998;10:198-202.

[23] Mamas MA, Eichhofer J, Hendry C, et al. Use of the Heartrail II catheter as a distal stent delivery device; an extended case series. EuroIntervention 2009;5:265-71.

[24] Zanchetta M, Pedon L, Rigatelli G, Olivari Z, Zennaro M, Maiolino P. Pseudo-lesion of internal mammary artery graft and left anterior descending artery during percutaneous transluminal angioplasty--a case report. Angiology 2004;55:459-62.

[25] Wong P, Rubenstein M, Inglessis I, Pomerantsev E, Ferrell M, Leinbach R. Spontaneous spiral dissection of a LIMA-LAD bypass graft: a case report. J Interv Cardiol 2004; 17:211-3. 
[26] Cantor WJ, Lazzam C, Cohen EA, et al. Failed coronary stent deployment. Am Heart J 1998;136:1088-95.

[27] Mamas MA, Fath-Ordoubadi F, Fraser DG. Telescoping catheter technique: looking towards the kidneys. Cardiovasc Revasc Med 2011;12:123-8.

[28] Farooq V, Mamas MA, Fath-Ordoubadi F, Fraser DG. The use of a guide catheter extension system as an aid during transradial percutaneous coronary intervention of coronary artery bypass grafts. Catheter Cardiovasc Interv 2011;78:847-63.

[29] Luna M, Papayannis A, Holper EM, Banerjee S, Brilakis ES. Transfemoral use of the GuideLiner catheter in complex coronary and bypass graft interventions. Catheter Cardiovasc Interv 2012;80:437-46.

[30] D'Amario D, Galli M, Vergallo R, Crea F, Porto I. How deep is your lesion? Extreme guideliner V3 intubation through RIMA graft to treat a distal left anterior descending artery stenosis. J Cardiovasc Med (Hagerstown) 2018;19:606-8.

[31] Park CI, Noble S, Bonvini RF. Guideliner microcatheter to improve back-up support during a complex coronary stenting procedure through a tortuous left internal mammary graft. J Invasive Cardiol 2012;24:E77-9.

[32] Cho SY, Moon KW, Kim JH, et al. The "Five-in-Seven" Guiding Technique to Overcome Proximal Coronary Tortuosity During Primary Percutaneous Coronary Intervention. Korean Circ J 2010;40:421-2. 